\title{
THE NUMBER OF FUNCTIONS DEFINING INTERPOLATING VARIETIES
}

\author{
SHIGEKI OH'UCHI
}

\begin{abstract}
In this paper, we prove that if a disjoint union of a countable number of complex affine subspaces is interpolating for the Hörmander algebra, then it can be written as the common zero set of $\alpha+1$ functions in the Hörmander algebra, where $\alpha$ is the maximum number of codimensions of the complex affine subspaces. Finally, we prove with an example in one complex variable that the number $\alpha+1$ is lowest.
\end{abstract}

\section{Introduction}

Let $X_{v}(v \in N$, the set of positive integers $)$ be $k_{v}$-codimensional complex affine subspaces of $C^{n}\left(1 \leq k_{v} \leq n\right)$, and put $\alpha=\max _{v \in N} k_{v}$. Assume that $X_{v} \cap$ $X_{v^{\prime}}=\emptyset$ for $v \neq v^{\prime}$. Let $N_{v}$ be the orthogonal linear subspaces of $X_{v}$, where we use the canonical inner product $\langle z, w\rangle=\sum_{l=1}^{n} z_{l} \bar{w}_{l}$ on $C^{n}$. Set $S_{v}=N_{v} \cap S^{2 n-1}$, where $S^{2 n-1}=\left\{u \in C^{n}:|u|=1\right\}$. Then Oh'uchi $[\mathrm{O}]$ proved the following result:

Theorem A. Let $X=\bigcup_{v \in N} X_{v}$ be an analytic subset of $\boldsymbol{C}^{n}$ consisting of disjoint complex affine subspaces $X_{v}$. Let $p$ be a weight function on $\boldsymbol{C}^{n}$. Then $X$ is interpolating for $A_{p}\left(\boldsymbol{C}^{n}\right)$ if and only if there exist $f_{1}, \ldots, f_{m} \in A_{p}\left(\boldsymbol{C}^{n}\right)(m \geq \alpha)$ and constants $\varepsilon, C>0$ such that

$$
X \subset Z\left(f_{1}, \ldots, f_{m}\right)\left(:=\left\{z \in \boldsymbol{C}^{n}: f_{1}(z)=\cdots=f_{m}(z)=0\right\}\right)
$$

and

$$
\sum_{j=1}^{m}\left|D_{u} f_{j}(\zeta)\right| \geq \varepsilon \exp (-C p(\zeta))
$$

for all $u \in S_{v}, \zeta \in X_{v}$ and $v \in N$.

Here the directional derivative $D_{u} f$ with a vector $u=\left(u_{1}, \ldots, u_{n}\right) \in S^{2 n-1}$ is defined by

This reserch is supported by JSPS Research Fellowships for Young Scientists.

Received March 15, 2000. 


$$
D_{u} f=\sum_{l=1}^{n} \frac{\partial f}{\partial z_{l}} \cdot u_{l} .
$$

For the terminologies, see $\S 2$. It extends the result of Berenstein and Li [BL1, Theorem 2.5], which deals with the case of $k_{v}=n$ for all $v \in N$.

Here we would like to discuss how many functions in $A_{p}\left(\boldsymbol{C}^{n}\right)$ we need to have an equality in (1.1). The main result of this paper is as follows:

Main Theorem. Let $X=\bigcup_{v \in N} X_{v}$ be an analytic subset of $\boldsymbol{C}^{n}$ consisting of disjoint complex affine subspaces $X_{v}$. Let $p$ be a weight function on $\boldsymbol{C}^{n}$. Then $X$ is interpolating for $A_{p}\left(\boldsymbol{C}^{n}\right)$ if and only if there exist $f_{1}, \ldots, f_{\alpha+1} \in A_{p}\left(\boldsymbol{C}^{n}\right)$ and constants $\varepsilon, C>0$ such that

$$
X=Z\left(f_{1}, \ldots, f_{\alpha+1}\right)
$$

and

$$
\sum_{j=1}^{\alpha+1}\left|D_{u} f_{j}(\zeta)\right| \geq \varepsilon \exp (-C p(\zeta))
$$

for all $u \in S_{v}, \zeta \in X_{v}$ and $v \in N$.

In $\S 4$, we prove that the number $\alpha+1$ is lowest by an example in one complex variable.

\section{Preliminaries}

We fix the notation. A plurisubharmonic function $p: \boldsymbol{C}^{n} \rightarrow[0, \infty)$ is called a weight function if it satisfies

$$
\log \left(1+|z|^{2}\right)=O(p(z))
$$

and there exist constants $C_{1}, C_{2}>0$ such that for all $z, z^{\prime}$ with $\left|z-z^{\prime}\right| \leq 1$

$$
p\left(z^{\prime}\right) \leq C_{1} p(z)+C_{2} .
$$

Definition 2.1. Let $\mathcal{O}\left(\boldsymbol{C}^{n}\right)$ be the ring of all entire functions on $\boldsymbol{C}^{n}$ and let $p$ be a weight function on $\boldsymbol{C}^{n}$. Set

$$
\begin{aligned}
& A_{p}\left(\boldsymbol{C}^{n}\right)=\left\{f \in \mathcal{O}\left(\boldsymbol{C}^{n}\right): \text { There exist constants } A, B>0\right. \text { such that } \\
&\left.|f(z)| \leq A \exp (B p(z)) \text { for all } z \in \boldsymbol{C}^{n}\right\} .
\end{aligned}
$$

Then $A_{p}\left(\boldsymbol{C}^{n}\right)$ is a subring of $\mathcal{O}\left(\boldsymbol{C}^{n}\right) . \quad A_{p}\left(\boldsymbol{C}^{n}\right)$ is often called the Hörmander algebra. The following lemma is easily deduced from (2.1) and (2.2):

Lemma 2.2. Let $p$ be a weight function on $\boldsymbol{C}^{n}$. Then the followings hold: (1) $\boldsymbol{C}\left[z_{1}, \ldots, z_{n}\right] \subset A_{p}\left(\boldsymbol{C}^{n}\right)$. 
(2) If $f \in A_{p}\left(\boldsymbol{C}^{n}\right)$, then $\partial f / \partial z_{j} \in A_{p}\left(\boldsymbol{C}^{n}\right)$ for $j=1, \ldots, n$.

(3) $f \in \mathcal{O}\left(\boldsymbol{C}^{n}\right)$ belongs to $A_{p}\left(\boldsymbol{C}^{n}\right)$ if and only if there exists a constant $K>0$ such that

$$
\int_{C^{n}}|f|^{2} \exp (-K p) d \lambda<\infty
$$

where $d \lambda$ denotes the Lebesgue measure on $\boldsymbol{C}^{n}$.

For the proof, see e.g. $[\mathrm{H}]$.

Example 2.3. (1) If $p(z)=\log \left(1+|z|^{2}\right)$, then $A_{p}\left(\boldsymbol{C}^{n}\right)=\boldsymbol{C}\left[z_{1}, \ldots, z_{n}\right]$.

(2) If $p(z)=|z|^{a}(a>0)$, then $A_{p}\left(C^{n}\right)$ is a space of entire functions which are of order $=a$ and of finite type, or which are of order $<a$.

(3) If $p(z)=|\operatorname{Im} z|+\log \left(1+|z|^{2}\right)$, then $A_{p}\left(\boldsymbol{C}^{n}\right)=\hat{\mathscr{E}}^{\prime}\left(\boldsymbol{R}^{n}\right)$, that is, the space of Fourier transforms of distributions with compact support on $\boldsymbol{R}^{n}$ (see e.g. [E]).

(4) When $p(z)=\exp |z|^{a}(a>0), p$ is a weight function if and only if $a \leq 1$.

In the rest of this paper, $p$ will always represent a weight function.

Definition 2.4. Let $X$ be an analytic subset of $\boldsymbol{C}^{n}$, and let $\mathcal{O}(X)$ be the space of analytic functions on $X$. Then we define

$$
\begin{aligned}
A_{p}(X)= & \{f \in \mathcal{O}(X): \text { There exist constants } A, B>0 \text { such that } \\
& |f(z)| \leq A \exp (B p(z)) \text { for all } z \in X\} .
\end{aligned}
$$

DeFINITION 2.5. An analytic subset $X$ in $C^{n}$ is said to be interpolating for $A_{p}\left(\boldsymbol{C}^{n}\right)$ if the restriction map $R_{X}: A_{p}\left(\boldsymbol{C}^{n}\right) \rightarrow A_{p}(X)$ defined by $R_{X}(f)=\left.f\right|_{X}$ is surjective.

The semilocal interpolating theorem by $[\mathrm{BT}]$ is useful to show an analytic subset to be interpolating. Let $X$ be given by

$$
X=Z\left(f_{1}, \ldots, f_{N}\right)=\left\{z \in C^{n}: f_{1}(z)=\cdots=f_{N}(z)=0\right\}
$$

with $f_{1}, \ldots, f_{N} \in A_{p}\left(C^{n}\right)$. Then for $\varepsilon, C>0$, we define

$$
S_{p}(f ; \varepsilon, C)=\left\{z \in C^{n}:|f(z)|=\left(\sum_{j=1}^{N}\left|f_{j}(z)\right|^{2}\right)^{1 / 2}<\varepsilon \exp (-C p(z))\right\},
$$

which is an open neighborhood of $X$. We recall the semilocal interpolation theorem of $[\mathrm{BT}]$.

Semilocal Interpolation Theorem. Let $h$ be a holomorphic function in $S_{p}(f ; \varepsilon, C)$ such that

$$
|h(z)| \leq A_{1} \exp \left(B_{1} p(z)\right)
$$

for all $z \in S_{p}(f ; \varepsilon, C)$, where $\varepsilon, C>0$. Then there exist an entire function 
$H \in A_{p}\left(C^{n}\right)$, constants $\varepsilon_{0}, C_{0}, A, B>0$ and holomorphic functions $g_{1}, \ldots, g_{N}$ in $S_{p}\left(f ; \varepsilon_{0}, C_{0}\right)$ such that

$$
H(z)-h(z)=\sum_{j=1}^{N} g_{j}(z) f_{j}(z)
$$

and

$$
\left|g_{j}(z)\right| \leq A \exp (B p(z))
$$

for all $z \in S_{p}\left(f ; \varepsilon_{0}, C_{0}\right)$ and $j=1, \ldots, N$. In particular, $H=h$ on the variety $X=Z\left(f_{1}, \ldots, f_{N}\right)$.

\section{The proof of the main theorem}

The sufficiency is included in Theorem A. Then we show the necessity.

Let $X=\bigcup_{v \in N} X_{v}$ be an analytic subset of $C^{n}$ consisting of disjoint complex affine subspaces $X_{v}$ of codimension $k_{v}$. Put $\alpha=\sup _{v \in N} k_{v}$. Then we define $f_{1}, \ldots, f_{\alpha} \in A_{p}\left(\boldsymbol{C}^{n}\right)$ by the following lemma, which follows from the proof of the necessity part of the main theorem in [O, pp. 377-384].

Lemma 3.1. If $X$ is interpolating for $A_{p}\left(C^{n}\right)$, then there exist $\alpha$ entire functions $f_{1}, \ldots, f_{\alpha} \in A_{p}\left(\boldsymbol{C}^{n}\right)$ and constants $\varepsilon, C>0$ such that

$$
X \subset Z\left(f_{1}, \ldots, f_{\alpha}\right)
$$

and

$$
\sum_{j=1}^{\alpha}\left|D_{u} f_{j}(\zeta)\right| \geq \varepsilon \exp (-C p(\zeta))
$$

for all $u \in S_{v}, \zeta \in X_{v}$ and $v \in N$.

Next we shall give $f_{\alpha+1}$. To do it, we need the following lemma:

Lemma 3.2. Let $f_{1}, \ldots, f_{\alpha} \in A_{p}\left(\boldsymbol{C}^{n}\right)$ be in Lemma 3.1. Let $\left\{X_{v}\right\} \cup\left\{Y_{\mu}\right\}$ be the set of all connected components of $Z\left(f_{1}, \ldots, f_{\alpha}\right)$. Then there exist constants $\varepsilon_{0}, C_{0}>0$ such that

$$
\sharp\left\{v \in N: X_{v} \cap W \neq \emptyset\right\} \leq 1
$$

for every connected component $W$ of $S_{p}\left(f ; \varepsilon_{0}, C_{0}\right)$. Moreover, letting $W_{v}$ be the connected component of $S_{p}\left(f ; \varepsilon_{0}, C_{0}\right)$ including $X_{v}$, we have $W_{v} \cap Y_{\mu}=\emptyset$ for every $\mu$.

Proof. Fix $v \in N$ and $\zeta \in X_{v}$. For $j \in\{1, \ldots, \alpha\}$ and $u \in S_{v}$, consider the entire function 


$$
f_{j, u, \zeta}(w)=f_{j}(w u+\zeta), \quad w \in \boldsymbol{C} .
$$

Setting

$$
f_{j, u, \zeta}^{\prime}(0)=\left.\frac{d}{d w}\right|_{w=0} f_{j, u, \zeta},
$$

we have

$$
\sum_{j=1}^{\alpha}\left|f_{j, u, \zeta}^{\prime}(0)\right| \geq \varepsilon \exp (-C p(\zeta))
$$

by (3.2). Hence, for all $u \in S_{v}$ there exists $j_{u} \in\{1, \ldots, \alpha\}$ such that

$$
\left|f_{j_{u}, u, \zeta}^{\prime}(0)\right| \geq \frac{\varepsilon}{\alpha} \exp (-C p(\zeta)) .
$$

Put

$$
V_{u, \zeta}=\left\{w \in C: f_{j_{u}, u, \zeta}(w)=0\right\}
$$

and

$$
d_{u, \zeta}= \begin{cases}\min \left\{1, \operatorname{dist}\left(0, V_{u, \zeta} \backslash\{0\}\right)\right\}, & \text { if } V_{u, \zeta \backslash\{0\} \neq \emptyset,} \\ 1, & \text { otherwise. }\end{cases}
$$

Since $f_{j_{u}} \in A_{p}\left(\boldsymbol{C}^{n}\right)$, we have

$$
\left|f_{j_{u}}(z u+\zeta)\right| \leq A_{1} \exp \left(B_{1} p(z u+\zeta)\right)
$$

for some constants $A_{1}, B_{1}>0$ independent of $\zeta, u$ and $v$. Thus (2.2) implies that for $|w| \leq 1$

$$
\left|f_{j_{u}, u, \zeta}(w)\right| \leq A_{2} \exp \left(B_{2} p(\zeta)\right),
$$

where $A_{2}=A_{1} \exp \left(B_{1} C_{2}\right)$ and $B_{2}=B_{1} C_{1}$. Set

$$
g_{u, \zeta}(w)=\frac{f_{j_{u}, u, \zeta}(w)}{w} .
$$

Since $f_{j_{u}, u, \zeta}$ has a zero at $w=0$ of order one, $g_{u, \zeta}$ is an entire function on $\boldsymbol{C}$ and

$$
g_{u, \zeta}(0)=f_{j_{u}, u, \zeta}^{\prime}(0) \neq 0 \text {. }
$$

By (3.4), on $|w|=1$ we have

$$
\left|g_{u, \zeta}(w)\right|=\frac{\left|f_{j_{u}, u, \zeta}(w)\right|}{|w|}=\left|f_{j_{u}, u, \zeta}(w)\right| \leq A_{2} \exp \left(B_{2} p(\zeta)\right) .
$$

It follows from the Maximum Modulus Theorem that for $|w| \leq 1$

$$
\left|g_{u, \zeta}(w)\right| \leq A_{2} \exp \left(B_{2} p(\zeta)\right) .
$$

Then the entire function 


$$
G_{u, \zeta}(w)=\frac{g_{u, \zeta}(w)-g_{u, \zeta}(0)}{3 A_{2} \exp \left(B_{2} p(\zeta)\right)}
$$

satisfies that $G_{u, \zeta}(0)=0$ and $\left|G_{u, \zeta}(w)\right|<1$ for $|w| \leq 1$. The Schwarz Lemma implies that $\left|G_{u, \zeta}(w)\right| \leq|w|$ for $|w| \leq 1$. In particular, for $w_{0} \in V_{u, \zeta} \backslash\{0\}$ with $\left|w_{0}\right| \leq 1$, we obtain from (3.3) and (3.5)

$$
\begin{aligned}
\left|w_{0}\right| \geq\left|G_{u, \zeta}\left(w_{0}\right)\right| & =\left|\frac{g_{u, \zeta}(0)}{3 A_{2} \exp \left(B_{2} p(\zeta)\right)}\right|=\left|\frac{f_{j_{u}, u, \zeta}^{\prime}(0)}{3 A_{2} \exp \left(B_{2} p(\zeta)\right)}\right| \\
& \geq \varepsilon_{3} \exp \left(-C_{3} p(\zeta)\right),
\end{aligned}
$$

where $\varepsilon_{3}=\varepsilon / 3 A_{2} \alpha$ and $C_{3}=C+B_{2}$. Hence,

$$
d_{u, \zeta} \geq \varepsilon_{3} \exp \left(-C_{3} p(\zeta)\right)
$$

Now we need the following Borel-Carathèodory inequality, (cf., e.g., [BG]).

Borel-Carathèodory inequality. Let $h$ be a function which is holomorphic in a neighborhood of $|w| \leq R$ and has no zero in $|w|<R$. If $h(0)=1$ and $0 \leq|w| \leq$ $r<R$, then the following estimate follows:

$$
\log |h(w)| \geq-\frac{2 r}{R-r} \log \max _{|\omega|=R}|h(\omega)| .
$$

Since $g_{u, \zeta}(0) \neq 0$, we apply this inequality to $h(w)=g_{u, \zeta}(w) / g_{u, \zeta}(0), R=d_{u, \zeta}$ and $r=d_{u, \zeta} / 2$, to obtain

$$
\begin{aligned}
\log \left|\frac{g_{u, \zeta}(w)}{g_{u, \zeta}(0)}\right| & \geq-\frac{2 \cdot\left(d_{u, \zeta} / 2\right)}{d_{u, \zeta}-d_{u, \zeta} / 2} \log \max _{|\omega|=d_{u, \zeta}}\left|\frac{g_{u, \zeta}(\omega)}{g_{u, \zeta}(0)}\right| \\
& =-2 \log \max _{|\omega|=d_{u, \zeta}}\left|\frac{g_{u, \zeta}(\omega)}{g_{u, \zeta}(0)}\right|
\end{aligned}
$$

for $|w| \leq d_{u, \zeta} / 2$. Then it follows from (3.3), (3.5) and (3.6) that

$$
\begin{aligned}
\left|g_{u, \zeta}(w)\right| & \geq\left|g_{u, \zeta}(0)\right|\left(\max _{|\omega|=d_{u, \zeta}}\left|\frac{g_{u, \zeta}(\omega)}{g_{u, \zeta}(0)}\right|\right)^{-2} \\
& =\left|g_{u, \zeta}(0)\right|^{3}\left(\max _{|\omega|=d_{u, \zeta}}\left|g_{u, \zeta}(\omega)\right|\right)^{-2} \\
& \geq \varepsilon_{4} \exp \left(-C_{4} p(\zeta)\right),
\end{aligned}
$$

where $\varepsilon_{4}=\varepsilon^{3} / \alpha^{3} A_{2}^{2}$ and $C_{4}=3 C+2 B_{2}$. Let

$$
\hat{d}_{\zeta}=\varepsilon_{3} \exp \left(-C_{3} p(\zeta)\right)
$$

where $\varepsilon_{3}$ and $C_{3}$ are the same as in (3.7). Since $\hat{d}_{\zeta} \leq d_{u, \zeta}$ by (3.7), it follows from (3.8) that for $|w|=\hat{d}_{\zeta} / 2$ 


$$
\left|f_{j_{u}, u, \zeta}(w)\right|=\left|w g_{u, \zeta}(w)\right| \geq \varepsilon_{5} \exp \left(-C_{5} p(\zeta)\right),
$$

where $\varepsilon_{5}=\varepsilon_{3} \varepsilon_{4} / 2$ and $C_{5}=C_{3}+C_{4}$. Thus we have proved that for every $u \in$ $S_{v}$, there exists $j_{u} \in\{1, \ldots, \alpha\}$ such that

$$
\left|f_{j_{u}}(w u+\zeta)\right| \geq \varepsilon_{5} \exp \left(-C_{5} p(\zeta)\right)
$$

for $|w|=\hat{d}_{\zeta} / 2$. Hence we have

$$
\begin{aligned}
|f(w u+\zeta)| & =\left(\sum_{j=1}^{\alpha}\left|f_{j}(w u+\zeta)\right|^{2}\right)^{1 / 2} \\
& \geq\left|f_{j_{u}}(w u+\zeta)\right| \geq \varepsilon_{5} \exp \left(-C_{5} p(\zeta)\right) .
\end{aligned}
$$

Note that the constants $\varepsilon_{5}$ and $C_{5}$ are independent of $u, \zeta$ and $v$.

For arbitrary $v \in N$ and $\zeta \in X_{v}$, we consider a neighborhood

$$
U_{v, \zeta}=\left\{z=w u+\zeta \in\{\zeta\}+N_{v}:|w| \leq \frac{\hat{d}_{\zeta}}{2}, u \in S_{v}\right\}
$$

of $\zeta$ in $\{\zeta\}+N_{v}$. For all $z \in \partial U_{v, \zeta}$, there exists $u_{z-\zeta} \in S_{v}$ such that

$$
z=\frac{\hat{d}_{\zeta}}{2} u_{z-\zeta}+\zeta
$$

Then it follows from (3.9) that

$$
|f(z)|=\left|f\left(\frac{\hat{d}_{\zeta}}{2} u_{z-\zeta}+\zeta\right)\right| \geq \varepsilon_{5} \exp \left(-C_{5} p(\zeta)\right) .
$$

Let $\hat{V}_{v}$ be the component of $S_{p}\left(f ; \varepsilon_{5}, C_{5}\right)$ containing $X_{v}$. Then it is clear that

$$
\hat{V}_{v} \subset \bigcup_{\zeta \in X_{v}} U_{v, \zeta}
$$

by (3.10). Now we claim that for $\zeta^{\prime} \in X_{v^{\prime}}$ and $v^{\prime} \neq v$,

$$
\zeta^{\prime} \notin \bigcup_{\zeta \in X_{v}} U_{v, \zeta} \text {. }
$$

In fact, by $\zeta$ we denote the orthogonal projection of $\zeta^{\prime}$ to $X_{v}$, so that $\zeta^{\prime} \in$ $\{\zeta\}+N_{v}$. Then there exists $u_{\zeta^{\prime}-\zeta} \in S_{v}$ such that

$$
\zeta^{\prime}=\left|\zeta^{\prime}-\zeta\right| u_{\zeta^{\prime}-\zeta}+\zeta .
$$

(3.7) implies that

$$
\left|\zeta^{\prime}-\zeta\right| \geq d_{u_{\zeta^{\prime}-\zeta}} \geq \varepsilon_{3} \exp \left(-C_{3} p(\zeta)\right)=\hat{d}_{\zeta} .
$$

Hence $\zeta^{\prime} \notin U_{v, \zeta}$. For $\tilde{\zeta} \in X_{v} \backslash\{\zeta\}$, it is clear that $\zeta^{\prime} \notin U_{v, \tilde{\zeta}}$. This proves the lamma for $\varepsilon_{0}=\varepsilon_{5}$ and $C_{0}=C_{5}$. 
Proof of the main theorem. Define a holomorphic function $h$ in $S_{p}\left(f ; \varepsilon_{0}, C_{0}\right)$ by

$$
h(z)= \begin{cases}0, & \text { if } z \in W_{v} \\ 1, & \text { if } z \in S_{p}\left(f ; \varepsilon_{0}, C_{0}\right) \backslash \bigcup_{v \in N} W_{v} .\end{cases}
$$

Then we have

$$
|h(z)| \leq 1 \cdot \exp (1 \cdot p(z))
$$

for every $z \in S_{p}\left(f ; \varepsilon_{0}, C_{0}\right)$. Hence it follows from the semilocal interpolation theorem that there exists an entire function $H \in A_{p}\left(\boldsymbol{C}^{n}\right)$ such that $\left.H\right|_{Z\left(f_{1}, \ldots, f_{\alpha}\right)} \equiv$ $\left.h\right|_{Z\left(f_{1}, \ldots, f_{\alpha}\right)}$, that is,

$$
H(z)= \begin{cases}0, & \text { if } z \in X_{v}, \\ 1, & \text { if } z \in Z\left(f_{1}, \ldots, f_{\alpha}\right) \backslash \bigcup_{v \in N} X_{v} .\end{cases}
$$

It is clear that $\alpha+1$ functions $f_{1}, \ldots, f_{\alpha}, H \in A_{p}\left(\boldsymbol{C}^{n}\right)$ satisfy (1.3) and (1.4).

\section{The sharpness of $\alpha+1$}

Finally, we remark that the number ' $\alpha+1$ ' in the main theorem is lowest. We prove this remark by giving an example of an interpolating variety for $A_{p}(\boldsymbol{C})$ which can not write as the zero set of a function in $A_{p}(\boldsymbol{C})$, where $p(z)=|z|$. Let $X=\left\{\zeta_{v}\right\}_{v \in N}$ be a discrete variety in $\boldsymbol{C}$. Then Nevanlinna's counting function is defined as follows: $n(r, X)=\sharp\left\{v \in N:\left|\zeta_{v}\right| \leq r\right\}$ and

$$
N(r, X)=\int_{0}^{r} \frac{n(t, X)-n(0, X)}{t} d t+n(0, X) \log r .
$$

For $k \in N$ and $r>0$, we define

$$
B(r ; k: X)=\frac{1}{k} \sum_{0<\left|\zeta_{v}\right| \leq r}\left(\frac{1}{\zeta_{v}}\right)^{k}
$$

and for $r_{1}, r_{2}>0$

$$
B\left(r_{1}, r_{2} ; k: X\right)=B\left(r_{1} ; k: X\right)-B\left(r_{2} ; k: X\right) .
$$

Then the following proposition which gives the relationship between an entire function in $A_{p}(\boldsymbol{C})$ and its zero set is deduced by the Fourier series method by Rubel and Taylor $[\mathrm{RT}]$ :

Proposition 4.1 (cf. [RT, Theorem 5.2]). Let $p$ be a radical weight of finite order on $\boldsymbol{C}$, that is, it satisfies $p(z)=p(|z|)$ and

$$
\varlimsup_{r \rightarrow \infty} \frac{\log p(r)}{\log r}<\infty .
$$


Then there exists $f \in A_{p}(\boldsymbol{C})$ such that $X=Z(f)$ if and only if

(1) $X$ has finite $p$-density, that is, we have a constant $A>0$ satisfying

$$
N(r, X) \leq A p(r)
$$

for every $r>0$.

(2) $X$ is p-balanced, that is, there exists a constant $A>0$ such that

$$
\left|B\left(r_{1}, r_{2} ; k: X\right)\right| \leq \frac{A p\left(r_{1}\right)}{r_{1}^{k}}+\frac{A p\left(r_{2}\right)}{r_{2}^{k}}
$$

for all $r_{1}, r_{2}>0$ and $k \in N$.

Example 4.2. Put $X=\{v\}_{v \in N} \subset C$. Applying Theorem A (or [BL2, Corollary 3.5]) to $f(z)=\sin \pi z \in A_{|\cdot|}(\boldsymbol{C})$, we know that $X$ is interpolating for $A_{|\cdot|}(\boldsymbol{C})$. Hence it follows from the main theorem that $X$ can be written as the common zero set of two entire functions in $A_{|\cdot|}(\boldsymbol{C})$. We shall prove that $X$ can be written as the zero set of no entire function in $A_{|\cdot|}(\boldsymbol{C})$.

Put $k=1$ and $r_{2}=1 / 2$. Then calculating $|B(r, 1 / 2 ; 1: X)|$, we have

$$
\left|B\left(r, \frac{1}{2} ; 1: X\right)\right|=\sum_{v=1}^{[r]} \frac{1}{v},
$$

where $[r]$ is the greatest integer not greater than $r$. Hence there does not exist a constant $A>0$ such that

$$
\left|B\left(r, \frac{1}{2} ; 1: X\right)\right| \leq \frac{A|r|}{r}+\frac{A / 2}{1 / 2}=2 A
$$

for any $r>0$. Thus $X$ does not satisfy the condition (2) in Proposition 4.1, so that $X$ can be written as a zero set of no entire function in $A_{|\cdot|}(\boldsymbol{C})$.

Finally, the main theorem and this example lead to the following conjecture:

Conjecture. Let $X$ be an interpolating variety for $A_{p}\left(C^{n}\right)$ and let $\alpha$ be the maximum number of codimentions of all irreducible components of $X$. Then $X$ can be written as the common zero set of $\alpha+1$ entire functions in $A_{p}\left(\boldsymbol{C}^{n}\right)$.

\section{REFERENCES}

[BG] C. A. Berenstein and R. Gay, Complex Variables: An Introduction, Graduate Text in Math. 125, Springer, New York, 1991.

[BL1] C. A. BERENSTEIN AND B. Q. LI, Interpolating varieties for weighted spaces of entire functions in $\boldsymbol{C}^{n}$, Publications Matemàtiques, 38 (1994), 157-173.

[BL2] —, Interpolating varieties for spaces of meromorphic functions, J. Geom. Anal., 5 (1995), 1-48.

[BT] C. A. Berenstein AND B. A. TAYlor, Interpolation problem in $\boldsymbol{C}^{n}$ with applications to harmonic analysis, J. Analyse Math., 38 (1981), 188-254. 
[E] L. Ehrenpreis, Fourier Analysis in Several Complex Variables, Pure and Applied Mathematics 17, Wiley, New York, 1970.

[G] R. GunNIng, Introduction to Holomorphic Functions of Several Variables Volume 1, Wadsworth, California, 1990.

[H] L. Hörmander, Generators for some rings of analytic functions, Bull. Amer. Math. Soc., 73 (1967), 943-949.

[O] S. OH'UCHI, Disjoint unions of complex affine subspaces interpolating for $A_{p}$, Forum Math., 11 (1999), 369-384.

[RT] L. A. Rubel and B. A. Taylor, A Fourier series method for meromorphic and entire functions, Bull. Soc. Math. France, 96 (1968), 53-96.

Department of Mathematics

Tokyo Institute of Technology

2-12-1 Oh-OKayama, Meguro-Ku

TOKYO, 152-8551, JAPAN

E-mail: ouchi@math.titech.ac.jp 